\title{
Organometallics for Electronics
}



Organometallics produced and analysed by Billiton Precursors B.V." are tested by Epitaxial Products International Ltd. and other institutions ensuring products of consistent high performance. Certificates of Analysis and Performance are supplied with each batch.

\section{Typical Precursor Performance}

\begin{tabular}{|c|c|c|c|c|c|c|c|}
\hline Precursor & $\begin{array}{c}\text { Epitaxial layer } \\
\text { grown }\end{array}$ & Temp. ${ }^{\circ}$ C & Pressure, mbar & V/III & n(300K) & $\mu(300 K)$ & $\mu(77 K)$ \\
\hline TMGa & GaAs & 650 & 100 & 110 & $7 e 13(\mathrm{C}-\mathrm{V})$ & 8900 & 155,000 \\
\hline TMln & InP & 650 & 200 & 270 & $3 e 14(\mathrm{C}-\mathrm{V})$ & 4600 & 120,000 \\
\hline TMAl* $^{*}$ & Alo.25Ga0.75As & 760 & 950 & 50 & 2 el5(Hall) & 3200 & 6,500 \\
\hline
\end{tabular}

" FWHM (5K) of Bound Exciton: (Alo .10GaO $90 \mathrm{As}$ ) 4.0 meV: (Alo 25 Gao 75As) 4.5meV

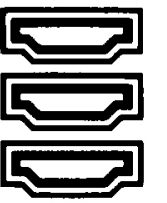

\section{Billiton Precursors B.V.}

Billiton Precursors B.V. * Dr R.G. Lythe, P.O. Box 639 (Westervoortsedijk 67d).6800 AP Arnhem. The Netherlands Tel (Int|)31-85-654882. Fax (IntI)31-85-654664. Telex 75026 bires $n \mid$
Billiton Metals Japan K. K. * Mr H Neriishi, Kasumigaseki Bldg $29 f$ 2-5. Kasumigaseki 3-chome. Chiyoda-Ku. Iokyo 100, Japan Tel (Int1)81-3-581-6303. Fax (Int) 81-3-592-0903. Telex j22373, j22901 
